\title{
Adatom density kinetic Monte Carlo: A hybrid approach to perform epitaxial growth simulations
}

\author{
L. Mandreoli* and J. Neugebauer \\ Fritz-Haber-Institut der Max-Planck-Gesellschaft, Faradayweg 4-6, D-14195 Berlin (Dahlem), Germany \\ R. Kunert and E. Schöll \\ Institut für Theoretische Physik, Technische Universität Berlin, Hardenbergstr. 36, D-10623 Berlin, Germany
}

(Received 19 February 2003; revised manuscript received 24 July 2003; published 29 October 2003)

We describe an alternative approach to perform growth simulations that combines the kinetic Monte Carlo (KMC) method with elements from continuum and rate equations. Similar to the KMC method it takes the atomistic structure of the growing surface fully into account but is based on the adatom density rather than on explicit trajectories of the adatoms. As will be demonstrated, this approach decouples the fast time scale of adatom motion from the much slower time scale of changes in growth morphology. This decoupling allows a reduction of the number of simulation time steps by several orders of magnitude. Based on a comparison with the KMC calculation performance, reliability and limits of this approach are discussed.

DOI: 10.1103/PhysRevB.68.155429

\section{INTRODUCTION}

Epitaxial growth is a key technique in fabricating semiconductor-based electronic and optoelectronic devices such as light-emitting diodes (LED's), laser diodes, or highelectron mobility transistors. These devices consist of vertically stacked thin films that differ by the material, alloy composition, or doping. To employ quantum effects some of these structures are only a few atomic layers thick. For the performance/efficiency of such devices the quality of the interfaces between the different layers is crucial. Realistic growth simulations could help to understand mechanisms affecting the interface quality but also to identify optimum growth conditions or suitable material combinations.

A challenge to perform such growth simulations is the large range of relevant length and time scales. The features interesting for device design (interface morphology, formation of nanostructures) are of the order of $10^{2}-10^{3} \mathrm{~nm}$ and the time to grow these structures is of the order of seconds. The origin of these effects, however, lies in the atomic processes on the surface (adatom adsorption, desorption, nucleation, etc.), which requires a resolution in the length scale $\approx 10^{-1} \mathrm{~nm}$ and in the time scale of $10^{-13} \mathrm{~s}^{-1}$.

Various approaches have been developed to overcome the scaling problem. In general, methods to perform growth simulations can be classified in three main categories: (i) rate equations (giving only global information such as island density or adatom coverage) without spatial resolution, (ii) continuum equations, which describe the surface morphology on a mesoscopic scale, and (iii) computer simulations, describing the full atomistic structure of the growing surface, such as molecular dynamics (MD) or the kinetic Monte Carlo (KMC) simulation. All of these methods had a tremendous effect on our understanding about various aspects of growth. However, all of them have certain deficiencies when applied to the above-mentioned topics: The first two approaches (i) and (ii) do not really bridge the large range of length and time scales but work exclusively on a mesoscopic scale by using effective parameters. A problem with these approaches is that the effective parameters cannot be directly related to the actual atomic parameters.

In MD simulations as input no a priori information is needed and they provide detailed insight into microscopic processes such as funneling and steering ${ }^{1,2}$ of deposition. But due to limitations in computational power, this method is mostly restricted to very short simulation times of the order of picoseconds, small simulation areas, and low temperatures. Even new MD approaches that separate time scales (e.g., atom-surface impact and adatom diffusion events) pertain only to very specific experimental setups ${ }^{3}$ where complex multiatom diffusion mechanisms were found. For a recent review on accelerated molecular dynamics methods see Ref. 4. These methods are important tools to identify all revevant diffusion process a priori and calculate their diffusion rates. However, so far they are computationally too expensive to describe large systems consisting of several $10^{4}-10^{5}$ atoms. Modeling crystal growth with the KMC method allows one to cover experimentally relevant growth times and system sizes, since each event on the surface is just described by a single quantity - the transition rate-rather than by modeling the full reaction path including atomic geometries and energies and the many (unsuccessful) attempts to realize it.

In the present paper we will derive a method that bridges the gap between the different categories of methods allowing fast growth simulations [such as (i) and (ii)] but using as input atomic processes (such as the KMC method). An advantage of this approach is that the input parameters are identical to those used in the KMC method, thus allowing a direct comparison between the two methods. Thus, it can be directly applied to any KMC method, in particular, it can be incorporated also into novel MD-based KMC methods (see, e.g., Ref. 5) that apply the dimer method (see Ref. 6) to efficiently calculate all kinetic parameters.

\section{METHOD}

\section{A. General}

In order to describe growth we must in principle follow the trajectory of each individual atom starting from the ad- 


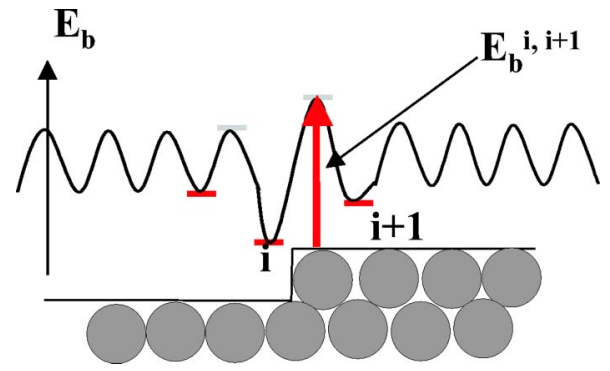

FIG. 1. One-dimensional potential energy surface (PES). $E_{b}^{i, i+1}$ is the energy barrier that one adatom has to overcome to hop from site $i$ to site $i+1$.

sorption on the surface over the motion on the surface until it eventually gets incorporated or desorbed. In general, all information necessary to get this information can be obtained by calculating the potential energy surface an atom experiences on a realistic surface. Realistic means here that the surface has various structural features such as surface steps, kinks, and facets. In principle, such a potential energy surface (PES) can be calculated based on first-principles total energy calculations (see, e.g., Ref. 7). Figure 1 shows a schematic example of such a PES for two terraces separated by a surface step. The site in front of the step has a higher coordination (the atom can form more bonds with the surface) and is thus energetically more favorable than the adsorption sites (local minima in the PES) on the flat surface. In the following we assign each of these minima (adsorption sites) an index $i$. To move the atom from one stable site $i$ to another site $i+1$ a barrier of height $E_{\text {diff }}^{i, i+1}$ has to be overcome. For further discussion we will assume that all barrier heights are much larger than the thermal energy of the adatom. This approximation is expected to be well fulfilled, since previous theoretical and experimental studies showed that diffusion barriers on semiconductor surfaces are of the order of a few tenths of an $\mathrm{eV}$ up to a few $\mathrm{eV} .{ }^{8}$ Under these conditions transition state theory applies and the transition rate for an adatom jump from site $i$ to $i+1$ is given by ${ }^{9,10}$

$$
w_{i \rightarrow i+1}=\Gamma_{i, i+1} \exp \left(-\frac{E_{\text {diff }}^{i, i+1}}{k_{B} T}\right) .
$$

Here, the prefactor $\Gamma$ is called attempt frequency and can be directly calculated from total energy calculations. $T$ is the temperature and $k_{B}$ the Boltzmann constant.

To describe surface growth we have not only to follow a single atom but an ensemble of atoms. Let certain configuration in this ensemble be $n$. As for the individual adatom each possible configuration is given by a minimum in the total energy surface and neighboring minima are separated by a barrier $E_{\text {diff }}^{n, n^{\prime}}$. Using these assumptions, performing a growth simulation requires us to find the sequence of configurations starting from an initial one (which corresponds to the initial surface). Mathematically, this is described by a master equation: ${ }^{11-16}$

$$
\frac{\partial P_{n}(t)}{\partial t}=\sum_{\left\{n^{\prime}\right\}}\left[w_{n^{\prime} \rightarrow n} P_{n^{\prime}}(t)-w_{n \rightarrow n^{\prime}} P_{n}(t)\right] .
$$

Here, $P_{n}(t)$ is the probability of finding the system at time $t$ in configuration $n$, and $w_{n^{\prime} \rightarrow n}$ is the transition rate to go from configuration $n$ to $n^{\prime}$. For a typical growth simulation these transitions involve adsorption, desorption, diffusion, and nucleation.

\section{B. Kinetic Monte Carlo method}

The most common approach to solve the above master equation for growth simulations is the kinetic Monte Carlo method. ${ }^{17-19}$ The main idea there is to start from a configuration $n_{0}$, calculate the transition probability $w_{n_{0} \rightarrow n^{\prime}}$ for all possible events, and select a new configuration by using a random number $r_{\text {rand }}$ in the interval between 0 and 1. Similarly, the time for this event can be calculated by ${ }^{20}$

$$
\Delta t=-\frac{\ln \left(r_{\text {rand }}\right)}{\sum_{n^{\prime}} w_{n_{0} \rightarrow n^{\prime}}} .
$$

$n^{\prime}$ runs over all neighbor configurations of $n_{0}$. The above procedure is subsequently repeated and one directly obtains how the growing surface evolves in time $n(t)$.

A problem with the KMC method is that with increasing growth temperature it becomes exceedingly expensive. The reason becomes obvious by inspecting Eq. (1) - the transition probability increases exponentially with temperature $T$. Since the transition probability is inversely proportional to the time step, Eq. (3), the number of time steps and thus the computational effort to follow the system over a fixed time $t$ increases exponentially.

To discuss this behavior in more detail let us be more specific and consider a characteristic KMC simulation. To calculate the diffusion barrier we use a simple linear bond cutting model:

$$
E_{\text {diff }}^{i, i+1}=E_{0}+n E_{b} .
$$

Here, $E_{0}$ is the energy of an atom on the free surface, $E_{b}$ is the binding energy the system gains when forming a bond, and $n$ is the number of neighbors. Specifically, we set $E_{0}$ $=1 \mathrm{eV}$ and $E_{b}=1 \mathrm{eV}$. We further use a surface where the adsorption sites are arranged on a square lattice. Periodic boundary conditions are assumed and the size of the surface unit cell consists of $100 \times 100$ sites. Further, a flux of 1 monolayer $(\mathrm{ML}) / \mathrm{s}$ and a total simulation time of $0.1 \mathrm{~s}$ have been assumed throughout.

Table I lists the resulting diffusion mean free path per atom and the average time between two diffusion events for temperatures varying between 500 and $1150 \mathrm{~K}$. As expected, the number of jumps an atom undergoes before it is incorporated increases exponentially and the time step between two jumps decreases exponentially. When looking at these numbers one should keep in mind that to perform a growth simulation we are not really interested in following the trajectory for each individual adatom but eventually only in changes in the growth morphology, i.e., if a new nucleus/island is formed on the surface or an adatom attaches to an existing island. The number of these events, however, is much 
TABLE I. Diffusion mean free path $\left(d_{\mathrm{MFP}}\right)$ per atom and average diffusion time step $\Delta t_{\text {diff }}$, for temperatures ranging between 500 and $1150 \mathrm{~K}, d_{\text {lat }}$ is the distance between nearest neighbor sites. The results have been obtained employing KMC on a $100 \times 100$ matrix (for more details see text).

\begin{tabular}{lcc}
\hline \hline Temperature $(\mathrm{K})$ & $d_{\mathrm{MFP}} /$ atom $\left(d_{\text {lat }}\right)$ & $\Delta t_{\text {diff }}(\mathrm{s})$ \\
\hline 500 & 30 & $3.3 \times 10^{-5}$ \\
750 & 470 & $2.1 \times 10^{-6}$ \\
1000 & 1800 & $5.6 \times 10^{-7}$ \\
1150 & 60000 & $1.7 \times 10^{-8}$ \\
\hline \hline
\end{tabular}

smaller and of the same order as the number of adsorbed adatoms (i.e., of the order of one per atom). For the specific simulation we have performed here, the total number of atoms reaching the surface is approximately 1000: The average time step between two growth events is thus of the order of $10^{-4} \mathrm{~s}$. As can be seen from Table I, for higher temperatures the time scale for the growth events (which we are eventually interested in) becomes orders of magnitude larger than that of the diffusion events. The question we will address in the following is whether and under what conditions it is possible to separate the two time scales.

\section{Adatom-probability kinetic Monte Carlo method}

In the following we will therefore derive an alternative approach to the KMC method for solving the master equation. To do this we will perform two steps: First, we derive a method that no longer calculates the complete trajectory of each adatom but rather the probability $\rho_{\alpha}(i, t)$, i.e., the probability to find adatom $\alpha$ at time $t$ on site $i$. We will call this method the adatom-probability KMC procedure (AP-KMC). In a second step we will no longer consider each individual probability but only the total adatom density $\rho(i, t)$ $=\Sigma_{\alpha} \rho_{\alpha}(i, t)$. This method will be called the adatom-density $\mathrm{KMC}$ procedure (AD-KMC) and is described in Sec. II D.

In order to separate adatom motion from surface morphology we formally rewrite the index $n$ describing a specific configuration in two parts: (i) the index $S$ describing the surface without the adatoms and (ii) the index $\left(i_{1}, i_{2}, \ldots, i_{N}\right)$, which gives the position of the adatoms on the surface:

$$
n \rightarrow\left\{\left(i_{1}, i_{2}, \ldots, i_{N}\right), S\right\} .
$$

$i_{\alpha}$ gives the site index of the $\alpha$ th adatom, and the total number of adatoms is $N$. In the following we describe the surface in a solid on solid (SOS) model by simply giving the height on each lattice site:

$$
S=\left\{h_{1}, h_{2}, \ldots, h_{M}\right\} .
$$

$M$ is the number of lattice sites. An extension to general surface structures is straightforward (e.g., by giving directly the atomic coordinates $\left\{R_{J}\right\}_{S}$ defining the surface $S$ ). The probability function then looks like

$$
P_{n}(t)=P_{a d}\left(i_{1}, i_{2}, i_{3}, \ldots, i_{N}, t\right) P_{S}(t) .
$$

Here, $P_{a d}\left(i_{1}, i_{2}, i_{3}, \ldots, i_{N}, t\right)$ is the probability for finding the adatoms in the configuration $\left(i_{1}, i_{2}, i_{3}, \ldots, i_{N}\right)$ at time $t$ and $P_{S}(t)$ is the probability for finding the surface in the configuration $S$ at time t. The advantage of this separation is that all the fast events (which are related to small activation barriers), are in $P_{a d}$, and all the slow events (in which, however, we are interested) are in $P_{S}$. For the typical time scale for growth, $\Delta t_{\text {growth }}$, the surface does not change. This means that $P_{S}=1$ and we can take it out of the master equation. Then, the master equation can be simplified. To see this, let us first formally rewrite the probability function:

$$
P_{n}(t)=P\left(i_{1}, i_{2}, i_{3}, \ldots, i_{N}, t\right) .
$$

If the adatoms do not interact, the probability function can be factorized in single adatom probability functions:

$$
P^{\text {nonint }}\left(i_{1}, i_{2}, i_{3}, \ldots, i_{N}, t\right)=\rho_{1}\left(i_{1}, t\right) \rho_{2}\left(i_{2}, t\right) \cdots \rho_{N}\left(i_{N}, t\right)
$$

and the master equation, Eq. (2), decomposes into a set of single adatom equations:

$$
\frac{\partial \rho_{\alpha}(i, t)}{\partial t}=\sum_{\delta}\left[w_{i+\delta \rightarrow i} \rho_{\alpha}(i+\delta, t)-w_{i \rightarrow i+\delta} \rho_{\alpha}(i, t)\right] .
$$

Here, $w_{i+\delta \rightarrow i}$ is the transition probability of an adatom to go from site $i+\delta \rightarrow i$ and $\delta$ is an index that goes over all nearest-neighbor sites. The time evolution of each single adatom probability can then be calculated as follows: (i) it is zero before adsorption of adatom $\alpha$ at time $t_{\alpha}^{\text {des }}$; (ii) it is

$$
\rho_{\alpha}\left(i, t_{\alpha}^{\mathrm{ads}}\right)=\delta_{i, i_{\text {ads }}}
$$

where $\delta_{i, i_{\text {ads }}}$ is the Kronecker symbol and $i_{\text {ads }}$ the adsorption site. Then, (iii) it is determined by Eq. (10), and (iv) after desorption or attachment it becomes zero again.

To implement and discuss this method we make the following assumptions: We choose the adsorption time deterministically

$$
t_{\alpha}^{\text {ads }}=\alpha \Delta t_{\text {growth }},
$$

where $\Delta t_{\text {growth }}=1 / L^{2} F$ is the average time for an adsorption/ growth event. $F$ is the flux of incoming particles (in ML/s) and $L^{2}$ is the total number of adsorption sites on the surface. The transition probabilities $W_{i \rightarrow i+\delta}$ are chosen identical to those used for the KMC simulations (see Sec. II B). The one-particle equation, Eq. (10), is solved by a finite difference scheme:

$$
\frac{\partial \rho_{\alpha}(i, t)}{\partial t}=\frac{\rho_{\alpha}(i, t+\Delta t)-\rho_{\alpha}(i, t)}{\Delta t} .
$$

A typical choice is $\Delta t=\Delta t_{\text {growth }}$, i.e., the simulation time step is no longer limited by the diffusion time step $\Delta t_{\text {diff }}$, which is orders of magnitude smaller than $\Delta t_{\text {growth }}$ (see Sec. II B).

To discuss the time evolution in more detail let us consider a single adatom in front of a surface step. This is shown in Fig. 2 for a temperature of $500 \mathrm{~K}$ and in $0.03 \mathrm{~s}$ time 

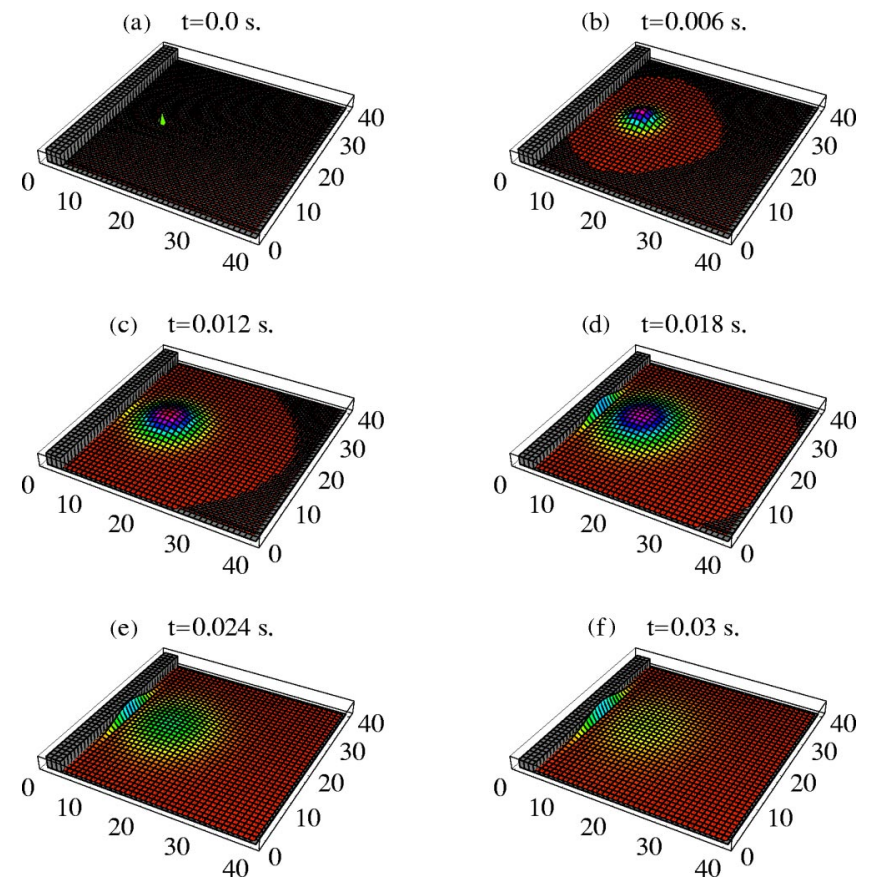

FIG. 2. Time evolution of the adatom density for an adatom close to a step. The snapshots are taken between $t=0.00 \mathrm{~s}$ and $t$ $=0.03 \mathrm{~s}$ for $T=500 \mathrm{~K}$. For $t>0$ the adatom density has been multiplied by an arbitrary scaling factor for ease of viewing.

intervals on an array of $40 \times 40$. At $t=0 \mathrm{~s}$ the adatom density is unity at the deposition site and zero everywhere else [see Eq. (11) and Fig. 2(a)]. In the next two time steps the adatom density spreads out on the surface [Figs. 2(b) and 2(c)] and eventually reaches the step. Since the probability to move away from the step is low-the barrier for jumping from the step to the terrace is $2 \mathrm{eV}$ compared to $1 \mathrm{eV}$ for the jump from the terrace to the step [see Eq. (4)] — the adatom density accumulates at the step edge [Figs. 2(d) and 2(e)]. In the last figure of this sequence the initial peak at the deposition site completely disappeared and almost all densities are concentrated along the step edge. We note that the density correctly describes all relevant processes: diffusion on the terrace, attachment and detachment at steps, and diffusion along the step.

It is important to note that the knowledge of the singleparticle densities $\rho_{\alpha}(i, t)$ allows us to obtain the position of the adatom at each time. To this end the single-particle density has to be collapsed on a single site, which can be done by choosing a random number $p_{\text {rand }}$ in the interval $[0,1]$ and selecting the site $l$ such that

$$
\sum_{i=0}^{l} \rho_{\alpha}(i, t) \leqslant r_{\text {rand }} \leqslant \sum_{i=0}^{l+1} \rho_{\alpha}(i, t) .
$$

Note that the integrated density is

$$
\bar{\rho}_{\alpha}=\sum_{i=0}^{L^{2}} \rho_{\alpha}(i, t) \equiv 1 .
$$
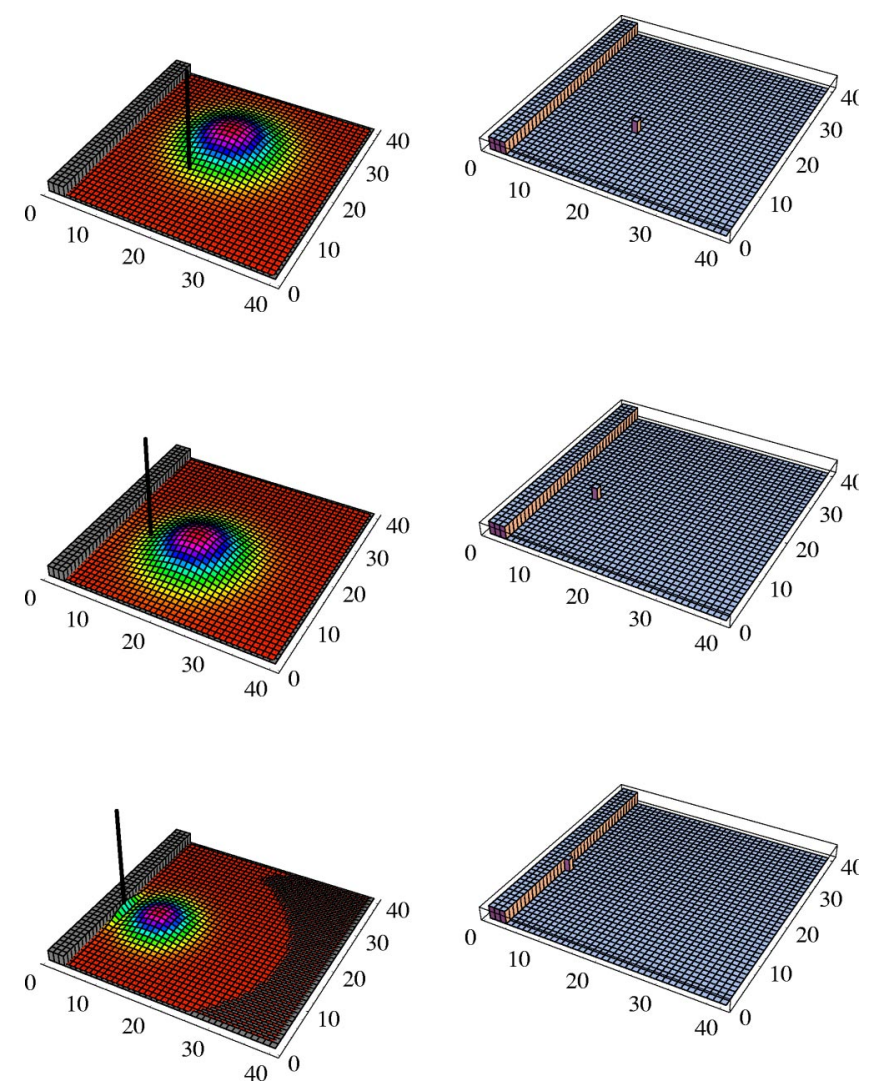

FIG. 3. Surface before (left) and after (right) a collapse of the adatom density. The collapse is performed at equal time intervals of $\Delta t=0.01 \mathrm{~s}$. The simulation has been performed at a temperature of $T=500 \mathrm{~K}$. The adatom density has been scaled for ease of viewing.

It should be noted that the collapse is not a deterministic process but stochastic in analogy to a measurement process of a quantum mechanical wave function. To be more specific, let us again consider the evolution of the single-particle density of an adatom in front of a surface step. This is shown in Fig. 3. The simulations were done at a temperature of $500 \mathrm{~K}$ and the collapse took place after a time interval of $\Delta t$ $=0.01 \mathrm{~s}$. As can be seen the adatom attaches to the step already after three collapses/simulation steps. Using the KMC method, where each individual jump is described, a much larger number of time steps would have been required (for the specific case shown in Fig. 3, approximately 800 steps), i.e., this approach indeed allows us to dramatically reduce the number of time steps.

Let us now generalize this method to take also interactions between adatoms into account. In the following we will assume that only adatoms on nearest-neighbor sites interact with each other. This would be, e.g., the case if adatoms on neighboring sites form a strong chemical bond so that longrange interactions due to elastic and/or electrostatic interactions can be neglected. For the simplified energy functional we use here, Eq. (4), this assumption is always fulfilled.

Since interaction is restricted between adatoms on neighboring sites it means that once an interaction occurs, a cluster/nucleus consisting of two or more adatoms on the surface is formed. Depending on the growth parameters (temperature, fluxes) the minimum size of such a cluster to 
form a stable nucleus may vary (see, e.g., Ref. 21). In order to keep the following discussion simple, we assume that already a nucleus consisting of two atoms is stable and will result in the formation of an island. A generalization to larger critical island sizes is straightforward and will be described elsewhere. ${ }^{22}$

Based on the above discussion, adatom-adatom interactions can be described by including nucleation events in the noninteracting adatom-adatom system. Following Eq. (10) the nucleation probability $p_{\alpha, \beta}(i, j, t)$ of adatom $\alpha$ to nucleate with adatom $\beta$ on neighboring sites $i$ and $j$ at time $t$ can be calculated from the single-particle densities $\rho_{\alpha}(i, t)$, $\rho_{\beta}(i, t)$ by

$$
\begin{aligned}
p_{\alpha, \beta}(i, j, t)= & \sum_{\delta_{i}} w_{i+\delta_{i} \rightarrow i} \rho_{\alpha}\left(i+\delta_{i}, t\right) \rho_{\beta}(j, t) \\
& +\sum_{\delta_{j}} w_{j+\delta_{j} \rightarrow j} \rho_{\alpha}(i, t) \rho_{\beta}\left(j+\delta_{j}, t\right) .
\end{aligned}
$$

$\delta_{i}$ and $\delta_{j}$ describe neighboring sites around sites $i$ and $j$.

To perform a growth simulation including adatom-adatom interactions is then straightforward and very similar to solving the noninteracting adatom-adatom system. The only difference is that in addition to solving Eq. (10) after each time step also all nucleation probabilities $p_{\alpha, \beta}(i, j, t)$ have to be calculated. Similarly as for the collapse of the single-particle densities we perform also a collapse for the nucleation density to decide whether and where a nucleation takes place. We therefore calculate the nucleation time by

$$
t_{\text {nuc }}=-\frac{\ln r_{\text {rand }}}{n_{\text {tot }}} .
$$

Here, $n_{\mathrm{tot}}=\frac{1}{2} \Sigma_{\alpha, \beta, i, j} p_{\alpha, \beta}(i, j, t)$ is the total probability of a nucleation event ( $j$ is restricted to be a nearest neighbor of $i)$. If $t_{\text {nuc }}$ is larger than the simulation time step $\Delta t_{\text {growth }}$, no nucleation event occurs. If, however, $t_{\text {nuc }}$ is smaller a nucleation event is performed by applying a further collapse [in analogy to Eq. (14)] to select the pair of atoms ( $\alpha$ and $\beta$ ) and the nucleation sites $i$ and $j$. On the site where the nucleation occurs the surface is updated, i.e.,

$$
S=\left(\ldots, h_{i}, h_{j}, \ldots\right) \Rightarrow S^{\prime}=\left(\ldots, h_{i}+1, h_{j}+1, \ldots\right) .
$$

The method will be applied and discussed in Sec. III.

\section{Adatom-density kinetic Monte Carlo method}

While the method described in the preceding section successfully decouples the "fast" time scale of the diffusion events from the "slow" time scale of the growth events, it is numerically still very expensive. The most expensive computations are those of the single-particle density and of the nucleation probability (which is a two-particle density and scales thus with the square of the number of adatoms).

In order to discuss further optimizations let us consider the case of high growth temperatures, which is particularly relevant for realistic growth simulations. Since the diffusivity (the jump probability) of an adatom increases exponentially with temperature at high temperatures, an adatom quickly explores a large part of the surface and gets quickly trapped on energetically favorable sites (such as surface steps or kinks). Under these conditions the total adatom density and thus the nucleation rate will be small. In other words, the one-particle density will be rather delocalized and will quickly "lose" the information about the initial adsorption site [see, e.g., Fig. 2(f)]. In the limit of infinite diffusivity each adatom can explore the entire surface, i.e., the singleparticle density will be infinite and describe the thermodynamic probability to find an adatom on a certain site. This probability function is of course identical for all adatoms. For conditions close to thermodynamic equilibrium the densities are not identical but similar.

If the one-particle densities are (at least in a local region) similar to each other and largely delocalized, the adatoms can be described by the total adatom density

$$
\rho(i, t)=\sum_{\alpha} \rho_{\alpha}(i, t)
$$

rather than by the complete set of single-particle densities. Using the same arguments as in the preceding section we then obtain a method called the AD-KMC method and that completely avoids the calculation of the single-particle densities. To keep the following discussion simple we assume aggregated growth, i.e., once an adatom has been incorporated it cannot be dislodged to become an adatom again. The equation describing the time evolution is similar to Eq. (10),

$$
\begin{aligned}
\frac{\partial \rho(i, t)}{\partial t}= & \sum_{\delta}\left[w_{i+\delta \rightarrow i} \rho(i+\delta, t)-w_{i \rightarrow i+\delta} \rho(i, t)\right] \\
& -n_{\text {nuc }}(i, t)-n_{\text {att }}(i, t)+F(i, t),
\end{aligned}
$$

except that nucleation $n_{\text {nuc }}(i, t)$, attachment $n_{\text {att }}(i, t)$ and adsorption $F(i, t)$ are now explicitly included. For the further discussion we will assume a constant and homogeneous flux $[F(i, t)=F]$. Attachment is described by a deterministic event, i.e., it occurs once the adatom density at site $i_{0}$ and at time $t_{0}$ is unity $\left[\rho\left(i_{0}, t_{0}\right)=1\right]$. If such an event occurs, $n_{\text {att }}(i, t)=\delta_{i, i_{0}} \delta_{t, t_{0}}$, i.e., the density on this site is reset to zero. Also, the surface and thus the transition probabilities $w_{i+\delta \rightarrow i}$ around the attached atom are modified:

$$
S=\left(\ldots, h_{i}, \ldots\right) \Rightarrow S^{\prime}=\left(\ldots, h_{i}+1, \ldots\right) .
$$

For a nucleation event on a terrace away from steps and for realistic growth parameters Eq. (16) can be further simplified. On a free terrace all transition rates are identical and given by the surface diffusion constant $D$ :

$$
w_{i+\delta_{i} \rightarrow i}=D / l_{0}^{2} .
$$

Here, $l_{0}$ is the distance between two nearest-neighbor sites. If we further assume that the adatom density around the nucleation site is approximately constant, we get the probability that a nucleation event takes place on site $i$ at time $t$ as

$$
p_{\text {nuc }}(i, t) \approx D l_{0}^{2} \rho^{2}(i, t),
$$


which is a well-known relation often applied in rate equations. $^{21}$ To calculate the nucleation rate $n_{\text {nuc }}(i, t)$ needed to solve Eq. (20) we assume:

$$
n_{n u c}(i, t) \equiv \bar{p}_{\text {nuc }}(t)=\int_{t-\Delta t_{\text {growth }}}^{t} \sum_{i} p_{\text {nuc }}(i, t) d t .
$$

The replacement of a localized nucleation term by a delocalized term is well justified for the high-temperature conditions assumed here and avoids the formation of localized regions with negative adatom density. For the modification of the surface and the transition states the nonaveraged nucleation term according to Eq. (23) is used. As can be seen from the above equations all quantities entering Eq. (20) are either explicitly given (like the flux $F$ ) or can be directly calculated from the total adatom density $\rho(i, t)$. Thus, in contrast to the AP-KMC method the explicit calculation of single-particle densities and two-particle nucleation terms is avoided, making the $\mathrm{AD}-\mathrm{KMC}$ method computationally much more efficient. Only for high temperatures do we expect the number of adatoms on the surface to be low, so the number of equations to solve (one for each adatom) will be small and the AP-KMC method can be almost as fast as the AD-KMC method. Applications of the method and a discussion about the validity of the underlying assumptions will be given in the next section.

\section{RESULTS FOR THE SUBMONOLAYER GROWTH}

To check the validity of the single and total adatom density-based approaches we performed an extensive comparison with the KMC method. For all three approaches identical simulation parameters have been chosen: The simulations have been performed on a square lattice (mesh size $80 \times 80)$, and the transition rates are calculated according to Eq. (1) using a prefactor $\Gamma=10^{13} \mathrm{~s}^{-1}$ and the diffusion barrier according to Eq. (4). ${ }^{23,24}$ The flux of incoming adatoms is $F=1 \mathrm{ML} / \mathrm{s}$. The total simulation time is $0.1 \mathrm{~s}$, i.e., a total of $0.1 \mathrm{ML}$ is deposited on the surface. We further assume that already a dimer forms a stable nucleus. This is explicitly enforced in the KMC calculations and allows us to approximate the nucleation in the AD-KMC simulation by Eq. (23). The growth temperature has been varied over a large range of temperatures (from 500 to $1000 \mathrm{~K}$ ). In order to analyze the results we have calculated and compared various statistical quantities such as island nucleation density, island size distribution, and island shape.

\section{A. Island nucleation density}

The first quantity that will be discussed is the island nucleation density, which is simply the number of islands formed on the surface. Rather than using directly the temperature as a variable we use the ratio of $D(T) / F$ as a free variable to allow a direct comparison with previous studies (see, e.g., Ref. 25). The calculated island nucleation density as function of $D / F$ is shown in Fig. 4. As can be seen, the $\mathrm{AD}-\mathrm{KMC}$ and $\mathrm{KMC}$ methods give virtually identical results: With increasing $D / F$ ratio, the island density rapidly decreases.

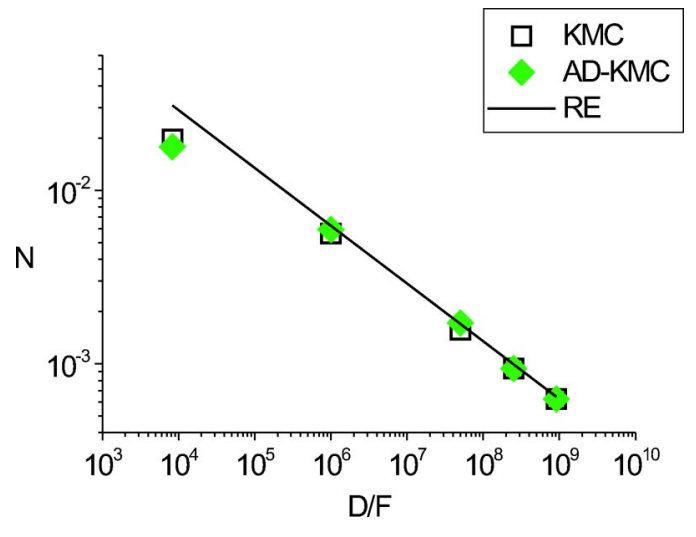

FIG. 4. Island density $N$ obtained with $\mathrm{AD}-\mathrm{KMC}$ and KMC methods as a function of $D / F$ between $10^{4}$ and $10^{9}$ at a coverage of $\theta=0.1 \mathrm{ML}$. The solid line (RE) is an analytic approximation based on rate equations and a critical nucleus size of two atoms (see text).

Employing rate equations the slope of this curve can be described analytically and is given by

$$
N \cong\left(\frac{D}{F}\right)^{-i /(i+2)}
$$

Here, $N$ is the island density, and $i$ gives the critical nucleus size, i.e., to be thermodynamically stable against dissociation a nucleus must consist of at least $i$ atoms. Since for our system already a dimer forms a stable nucleus $i=1$, the nucleation density should follow a scaling law with power $-1 / 3$. This scaling relation has been also included in Fig. 4. Since an analytical solution of the proportionality factor in the scaling relation, Eq. (25), is not known it has been fitted to the $\mathrm{KMC}$ result for $D / F=10^{9}$. As can be seen the scaling relation correctly approximates the slope of the $\mathrm{KMC}$ and $\mathrm{AD}-\mathrm{KMC}$ results except for very low $D / F$ ratios. Under these conditions the adatom diffusivity is negligible and nuclei are no longer formed by two adatoms moving together but by adsorption of an adatom next to an existing adatom.

\section{B. Island size distribution}

Having verified that the nucleation density is correctly described we will now focus on spatial information. To this

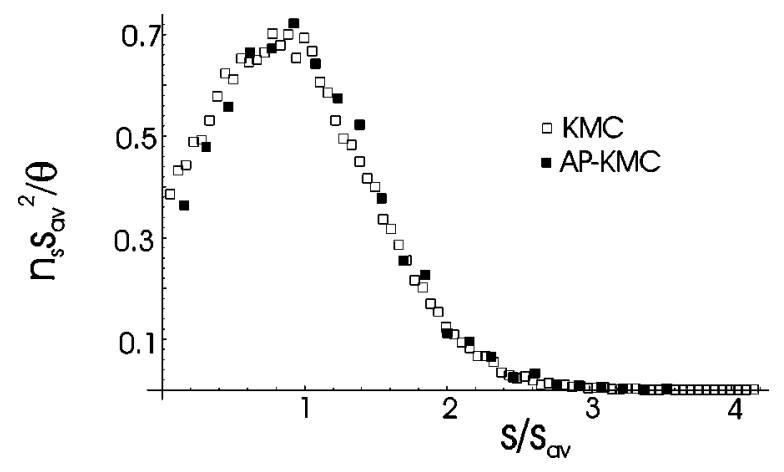

FIG. 5. Island size distribution obtained from KMC (filled squares) and AP-KMC (empty squares) methods for $T=630 \mathrm{~K}$, $D / F=10^{6}$, and with a coverage of $\theta=0.1 \mathrm{ML}$. Under these conditions fractal islands are formed (see Fig. 7). 


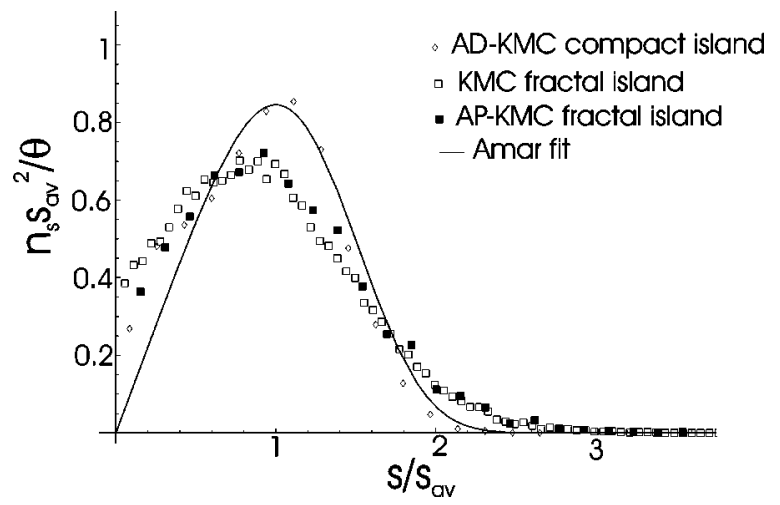

FIG. 6. Island size distribution as obtained by the KMC (empty squares), AP-KMC (filled squares), and AD-KMC (diamonds) methods for $T=630 \mathrm{~K}$ and a ratio $D / F=10^{6}$. The solid line is the universal scaling relation Eq. (26), as given in Ref. 25.

purpose we have calculated the island size distribution. Previous studies ${ }^{26}$ addressing this quantity showed that over a large range of fluxes, temperature, and diffusion barriers the island size distribution approximately follows a universal scaling law:

$$
n_{s}=\frac{\Theta}{s_{a v}^{2}} g_{i}\left(s / s_{a v}\right) .
$$

Here, $n_{s}$ is density of islands of size $s, s_{a v}$ is the average island size, and $g_{i}(u)$ is a scaling function with $i$ the critical nucleus size. Based on scaling laws Amar and Family ${ }^{25}$ gave an analytic fit for the scaling function $g_{i}(u)$ :

$$
g_{i}(u)=C_{i} u^{i} \exp \left(-i a_{i} u^{1 / a_{i}}\right) .
$$

Here, $a_{i}$ and $C_{i}$ are determined by

$$
\begin{gathered}
\frac{\Gamma\left((i+2) a_{i}\right)}{\Gamma\left((i+1) a_{i}\right)}=\left(i a_{i}\right)^{a_{i}}, \\
C_{i}=\frac{\left(i a_{i}\right)^{(i+1) a_{i}}}{a_{i} \Gamma\left((i+1) a_{i}\right)} .
\end{gathered}
$$

$\Gamma$ is the Gamma function.

As a first comparison between KMC and AP-KMC methods let us consider the island size distribution. A temperature $T=630 \mathrm{~K}$ has been chosen and the results are shown in Fig. 5. At this temperature both methods show the formation of fractal islands. The agreement is excellent, demonstrating the accuracy of the AP-KMC method. Figure 6 shows the island (a)

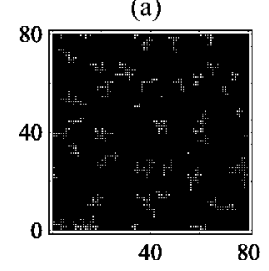

(b)

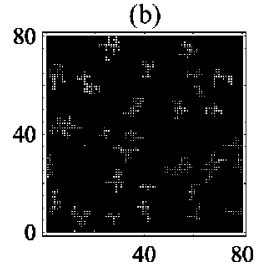

(c)

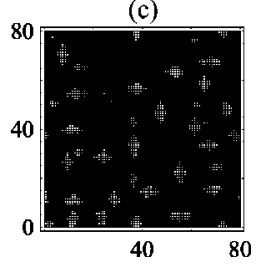

FIG. 7. Example of a growth simulation applying (a) KMC, (b) AP-KMC, and (c) AD-KMC methods for $630 \mathrm{~K}$.
$\mathrm{T}=1000 \mathrm{~K}$
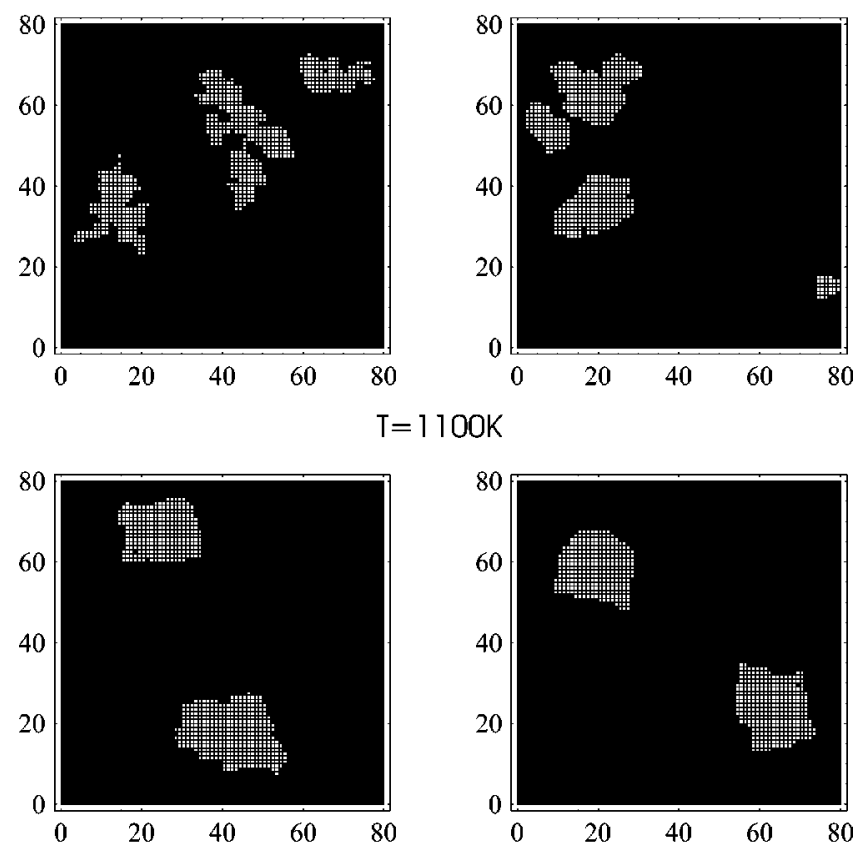

$\mathrm{T}=1200 \mathrm{~K}$
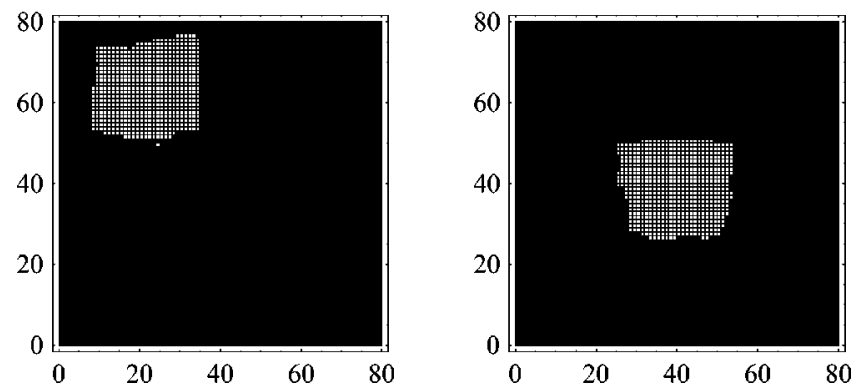

FIG. 8. Comparison of the island shape in KMC (left) and AD$\mathrm{KMC}$ (right) simulations for temperatures ranging from $1000 \mathrm{~K}$ to $1200 \mathrm{~K}$. Simulations have been performed on a $80 \times 80$ matrix.

size distribution for all three approaches. A total of 45 runs on a $300 \times 300$ matrix has been performed to obtain reliable statistics. As can be seen (see also Fig. 5) the island size distribution as calculated by KMC and AP-KMC methods is identical within the statistical error bars. However, the ADKMC method exhibits rather large deviations: The maximum is larger and shifted towards larger island sizes. Also, the distribution function is narrower than that for KMC and APKMC methods. An interesting behavior we obtain from Fig. 6 is that the asymptotic fit Eq. (27) describes the KMC and $\mathrm{AP}-\mathrm{KMC}$ results only rather poorly. This is not too surprising since these results are not yet in the asymptotic scaling limit of very large D/F. Interestingly, however, there is good agreement between the $\mathrm{AD}-\mathrm{KMC}$ results and this asymptotic expression.

In order to understand this puzzling behavior we have analyzed in more detail the morphology and shape of the islands. Figure 7 shows example surfaces for each of the three approaches. As can be seen both KMC and AP-KMC methods exhibit fractal growth, indicating that the flux ratio chosen here corresponds to rather low temperatures. The 

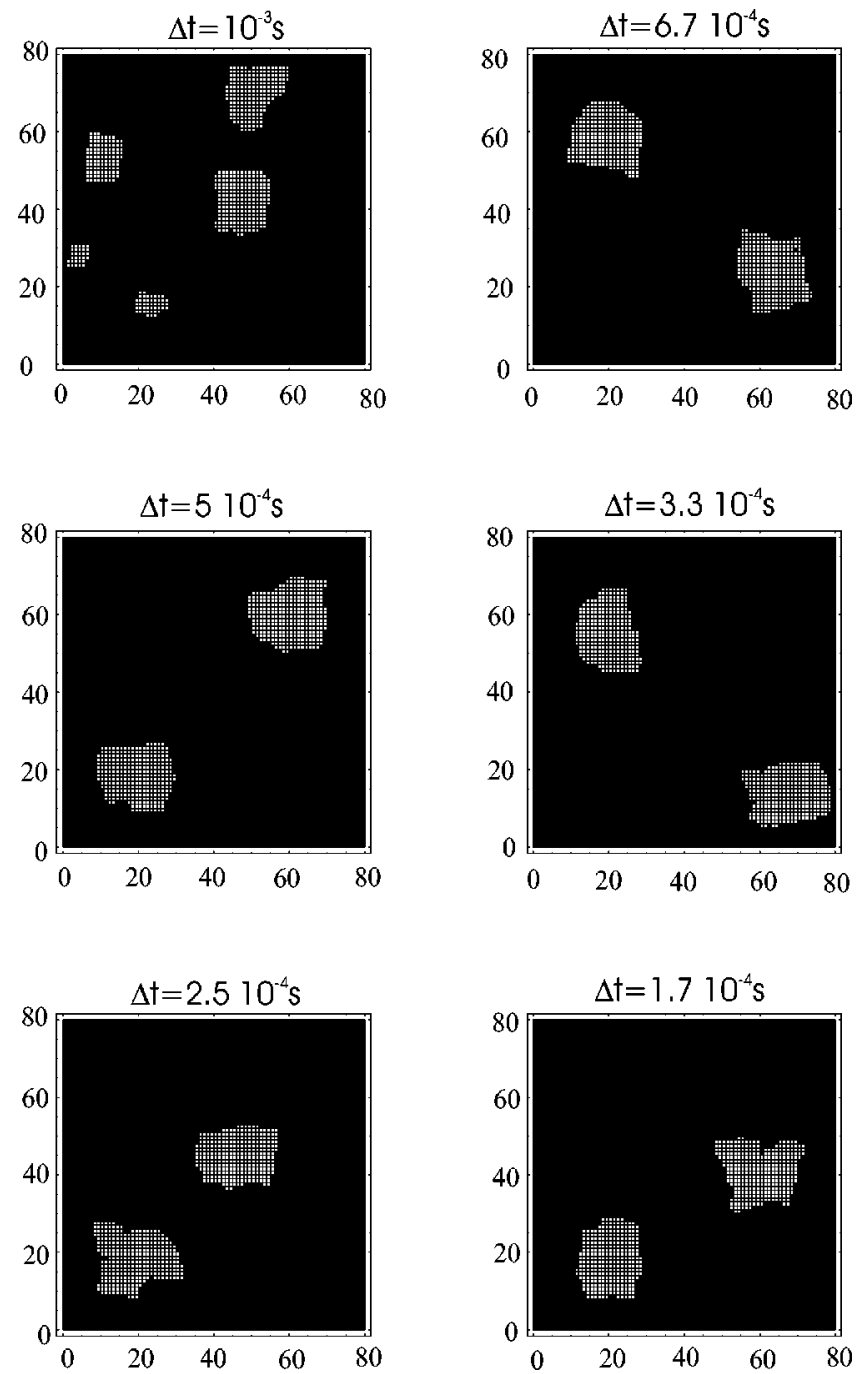

FIG. 9. AD-KMC simulations for $T=1100 \mathrm{~K}$ with different time steps $\Delta t=10^{-3}-1.7 \times 10^{-4} \mathrm{~s}$.

AD-KMC method shows a very different shape: islands are not fractal but more compact. The obvious failing of the $\mathrm{AD}-\mathrm{KMC}$ method is related to the fact that to derive this scheme we assumed that the system is close to thermodynamic equilibrium, i.e., temperatures are high enough to realize delocalized adatom densities. Based on the discrepancy in the island size distribution, but also in the island shapes (see Fig. 7) the temperature chosen here is much too low.

\section{Island shapes}

Let us now focus on a comparison of the different growth simulations at high temperatures. The calculations of the island size distribution with reliable statistics at high temperatures is computationally rather expensive and we will therefore focus on a discussion of the island shapes. Figure 8 shows characteristic surfaces as obtained from KMC and AD-KMC simulations for temperatures between 1000 and $1200 \mathrm{~K}$. For temperatures above $1100 \mathrm{~K}$ the agreement is excellent: Both methods show compact islands with similar features such as the density of kinks. Also, both methods

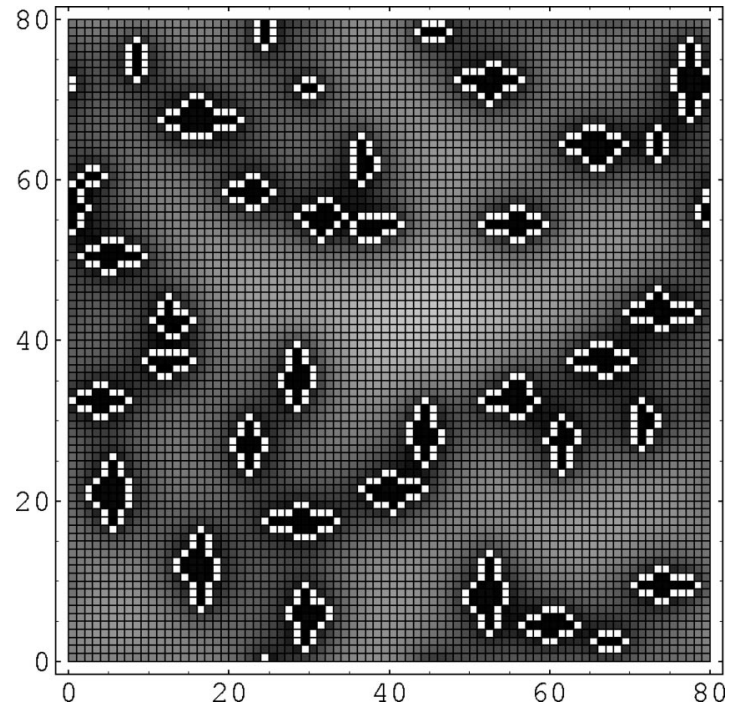

FIG. 10. Density plot of the adatom density. Dark regions mark surface areas with low density, bright regions those with high adatom density. The island borders are easily visible by a bright rim due to the enhanced adatom density. The dark regions around the islands are adatom-depleted areas (the adatoms have been captured by the islands). The density has been obtained from an AD-KMC calculation with $D / F=10^{6}$.

show that with increasing temperature the deviations from the equilibrium shape (which is a square for the parameters and lattice chosen here) become smaller. Only for the lowest temperature, where the KMC method shows the formation of fractal-like structures, the AD-KMC method gives islands too compact as already found for the low-temperature case. We can therefore conclude that for high temperatures (where the KMC method becomes exceedingly expensive) the ADKMC method is an efficient and accurate tool. Let us now check the sensitivity of the AD-KMC method to the choice of the discrete time step $\Delta t$. This time step determines after which time the adatom density is updated according to Eq. (20). For the AD-KMC results at $T=1100 \mathrm{~K}$ in Fig. 8 we used a time step $\Delta t=6.7 \times 10^{-4} \mathrm{~s}$. The typical growth time scale for this simulation is $\Delta t_{\text {growth }} \approx 1.6 \times 10^{-3} \mathrm{~s}$, so the time step $\Delta t$ is roughly a factor of 2 smaller than $\Delta t_{\text {growth }}$. In Fig. 9 we show AD-KMC simulations with the same growth parameters, but with $\Delta t$ ranging between $10^{-3}$ and 1.7 $\times 10^{-4} \mathrm{~s}$. As one can see, the simulations with smaller time steps $\Delta t=5 \times 10^{-4}-1.7 \times 10^{-4} \mathrm{~s}$ do not sensitively depend upon $\Delta t$. However, for a larger time step $\left(\Delta t=10^{-3} \mathrm{~s}\right)$ the number of islands is increased. The reason is that directly after a nucleation event the nucleus grows very quickly to a large island. In order to describe this process correctly the time step must be chosen small enough to resolve the process correctly. In the case considered here we find that the time step should be smaller than $1 / 2$ of the growth time step ( $\Delta t \leqslant \frac{1}{2} \Delta t_{\text {growth }}$ ). If it is chosen larger, the adatom density in the vicinity of the island is too large, leading to an enhanced nucleation.

\section{Capture zones}

An important quantity to analyze in growth simulations is the capture zone. The capture zone of an island defines the 
region around an island where adsorbing adatoms on the average diffuse to that island and are incorporated there. An interesting feature of the density-based KMC methods described in this paper is that they give direct insight into the capture zones. As an example let us discuss Fig. 10, which shows a characteristic adatom density as calculated by the AD-KMC method. Clearly visible are the adatom-depleted regions (dark areas) around all islands, which mark the capture zones. From the picture it becomes also clear that some capture zones coalesce (these islands compete for the same adatoms and will grow more slowly), while others are open to higher-density regions (those islands will grow faster).

\section{CONCLUSION}

We have introduced density-based KMC simulations that allow for an efficient separation of the fast time scale for the adatom kinetics from the much slower time scale describing changes in the surface morphology. In contrast to conventional KMC methods, where the characteristic time step is that of an adatom jump, the characteristic time step for the approach considered here is that of a growth event. Thus, particularly at high growth temperatures the number of simulation steps is reduced by orders of magnitude compared to the KMC method. An interesting feature of our approach compared to alternative approaches proposed for fast growth simulations (such as, e.g., the level set method ${ }^{27}$ ) is that it is directly linked to the microscopic structure and kinetics. This allows, e.g., a direct comparison between KMC and densitybased KMC simulations by using identical parameters. These tests show that for conditions far away from thermodynamic equilibrium (fractal growth) the adatom density for each adatom (AP-KMC) has to be calculated. For high temperature close to thermodynamic equilibrium (where the conventional KMC method becomes numerically exceedingly expensive) only the total density of all the adatoms is needed (AD$\mathrm{KMC}$ ). At these conditions, island nucleation density, island size distribution, and shape are correctly reproduced. From a numerical point of view the density-based approach has a number of advantages. Numerically, the main effort is in solving a diffusion equation, which has been extensively studied and for which efficient tools exist (e.g., multigrid schemes $^{28}$ ). Also in contrast to the KMC method where domain decomposition poses a severe problem for parallelization, the latter is straightforward for a diffusion problem.

\section{ACKNOWLEDGMENTS}

The project is financially supported by the EU TMR program "IPAM" under the reference number HPRN-CT-199900040 and by Sfb 296. We like to thank C. Ratsch (UCLA) for stimulating discussions.
*Electronic address: mandreoli@fhi-berlin.mpg.de

${ }^{1}$ J.W. Evans, D.E. Sanders, P.A. Thiel, and A.E. DePristo, Phys. Rev. B 41, 5410 (1990).

${ }^{2}$ F. Montalenti and A.F. Voter, Phys. Rev. B 64, 081401(R) (2001).

${ }^{3}$ F. Montalenti, M.R. Sørensen, and A.F. Voter, Phys. Rev. Lett. 87, 126101 (2001).

${ }^{4}$ A.F. Voter, F. Montalenti, and T.C. Germann, Annu. Rev. Mater. Sci. 32, 321 (2002).

${ }^{5}$ G. Henkelman and H. Jónsson, Phys. Rev. Lett. 90, 116101 (2003).

${ }^{6}$ G. Henkelman and H. Jónsson, J. Chem. Phys. 115, 9657 (2001).

${ }^{7}$ J. Neugebauer, T. Zywietz, M. Scheffler, and J. Northrup, Appl. Surf. Sci. 159, 355 (2000).

${ }^{8}$ T. Zywietz, J. Neugebauer, and M. Scheffler, Appl. Phys. Lett. 73, 487 (1998).

${ }^{9}$ V. Bortolani, N. March, and M. Tosi, Interaction of Atoms and Molecules with Solid Surfaces (Plenum Press, New York, 1990).

${ }^{10}$ J. Krug, Physica A 313, 47 (2002).

${ }^{11}$ A.C. Levi and M. Kotrla, J. Phys.: Condens. Matter 9, 299 (1997).

${ }^{12}$ Y. Saito, Statistical Physics of Crystal Growth (World Scientific, Singapore, 1996).

${ }^{13}$ D.D. Vvedensky, A. Zangwill, C.N. Luse, and M.R. Wilby, Phys. Rev. E 48, 852 (1993).

${ }^{14}$ M. Predota and M. Kotrla, Phys. Rev. E 54, 3933 (1996).
${ }^{15}$ A. Zangwill, C.N. Luse, D.D. Vvedensky, and M.R. Wilby, Surf. Sci. Lett. 274, L529 (1992).

${ }^{16}$ N. van Kampen, Stochastic Processes in Physics and Chemistry (North Holland, Amsterdam, 1981).

${ }^{17}$ D. Landau and K. Binder, A Guide to Monte Carlo Simulation in Statistical Physics (Cambridge University Press, Cambridge, 2000).

${ }^{18}$ M.E.J. Newman and G.T. Barkema, Monte Carlo Methods in Statistical Physics (Oxford University Press, Oxford, 2001).

${ }^{19}$ A.F. Voter, Phys. Rev. B 34, 6819 (1986).

${ }^{20}$ K.A. Fichthorn and W.H. Weinberg, J. Chem. Phys. 95, 1090 (1991).

${ }^{21}$ A. Pimpinelli and J. Villain, Physics of Crystal Growth (Cambridge University Press, Cambridge, 1998).

${ }^{22}$ L. Mandreoli and J. Neugebauer (unpublished).

${ }^{23}$ M. Meixner, E. Schöll, V.A. Shchukin, and D. Bimberg, Phys. Rev. Lett. 87, 236101 (2001).

${ }^{24}$ E. Schöll and S. Bose, Solid-State Electron. 42, 1587 (1998).

${ }^{25}$ J.G. Amar and F. Family, Phys. Rev. Lett. 74, 2066 (1995).

${ }^{26}$ A.-L. Barabasi and H. Stanley, Fractal Concepts in Surface Growth (Cambridge University Press, Cambridge, 1995).

${ }^{27}$ M.F. Gyure, C. Ratsch, B. Merriman, R.E. Caflisch, S. Osher, J.J. Zinck, and D.D. Vvedensky, Phys. Rev. E 58, R6927 (1998).

${ }^{28}$ W.H. Press, B.P. Flannery, S.A. Teukolsky, and W.T. Vetterling, Numerical Recipes in C: The Art of Scientific Computing (Cambridge University Press, Cambridge, 1998). 